\title{
Considering sarcoma staging systems and their implications to personalized care
}

\author{
Rick L. Haas ${ }^{1,2}$, Michiel A. J. van de Sande ${ }^{3}$ \\ ${ }^{1}$ Department of Radiotherapy, The Netherlands Cancer Institute, Amsterdam, The Netherlands; ${ }^{2}$ Department of Radiation Oncology, ${ }^{3}$ Department \\ of Orthopedic Surgery, The Leiden University Medical Center, Leiden, The Netherlands \\ Correspondence to: Rick L. Haas, MD, PhD. Department of Radiotherapy, The Netherlands Cancer Institute, Plesmanlaan 121, 1066 CX, Amsterdam, \\ The Netherlands. Email: r.haas@nki.nl. \\ Comment on: Johnson AC, Ethun CG, Liu Y, et al. A novel, simplified, externally validated staging system for truncal/extremity soft tissue sarcomas: \\ An analysis of the US Sarcoma Collaborative database. J Surg Oncol 2018;118:1135-41.
}

Submitted Dec 31, 2018. Accepted for publication Jan 10, 2019.

doi: $10.21037 /$ cco.2019.01.03

View this article at: http://dx.doi.org/10.21037/cco.2019.01.03

\section{Introduction}

Throughout oncology, the three most important (but not only) reasons to propose staging systems are:

(I) to speak the same language across the globe;

(II) to be able to compare outcome parameters between different hospitals;

(III) to predict outcome.

For most (epithelial) malignancies, the so-called TNM nomenclature described diseases with respect to tumor size $(\mathrm{T})$ and the presence of nodal $(\mathrm{N})$ and/or hematogenous $(\mathrm{M})$ metastases.

Daily clinical applicability of such systems and their use are dependent upon simplicity. Clinicians should preferably be able to remember the definitions by heart, without the need to consult elaborate books or websites. Hereto, for instance for many carcinomas, a $\mathrm{T} 1$ is $<2 \mathrm{~cm}$, a $\mathrm{T} 3>5 \mathrm{~cm}$, a $\mathrm{T} 2$ in between and a $\mathrm{T} 4$ with ingrowth into neighboring organs. This simplicity obviously harbors pitfalls; it disregards cytogenetic and molecular-biological predictive factors across tumors of the same size.

Recently, Johnson and co-workers have published a very interesting and easy novel staging system in the fournal of Surgical Oncology (1). They used their Sarcoma Collaborative database compiling results form 1,318 patients resected with curative intent between 2000 and 2016 across 7 tertiary referral centers. Patients with desmoid tumors, uterine sarcomas, and those who underwent $\mathrm{R} 2$ resection or died within 30 days of their operation were excluded as well as those histological subtypes that, by definition, should not be graded. In their proposed new staging system, all intermediate and high-grade tumors are lumped into the category of high grade. Stage I is designated for all lowgrade sarcomas regardless of size as long as they are not metastasized. Among stage II and III are patients with high grade tumors without any metastases; the dichotomy is on a size cut-off set at $7.5 \mathrm{~cm}$. In stage IV patients are diagnosed with any kind of metastases regardless of grade or size.

Authors have compared their patient outcomes to the American Joint Committee on Cancer (AJCC) staging systems, edition 7 and $8(2,3)$. In all three systems, among stage I cases are patients with non-metastasized low-grade tumors, where the AJCC systems further subdivide into suffix $\mathrm{A}$ and $\mathrm{B}$ on a cut-off of $5 \mathrm{~cm}$. In all three systems, metastatic disease is categorized to stage IV. The major difference among the three systems lies in the fact that the AJCC 8th edition dissects all non-metastatic highgrade tumors into $\leq 5 \mathrm{~cm}$ to stage II, $5-10 \mathrm{~cm}$ to stage IIIA and $\geq 10 \mathrm{~cm}$ as stage IIIB. The only difference in the new proposed system is a cut-off at $7.5 \mathrm{~cm}$ to be lumped into stage II or, if larger than $7.5 \mathrm{~cm}$ into stage III. The authors concluded that their proposed staging system maintained prognostic significance between stages though being a simplified system and, finally, that for high-grade tumors, a cutoff of $7.5 \mathrm{~cm}$, instead of $5 \mathrm{~cm}$, maintained discrimination for survival and could be a more clinically applicable cutoff for future clinical trials. As such, this system indeed fulfills the criterion of simplicity, perhaps even more than prior systems, but, as all others, lacks a personalized view and it 

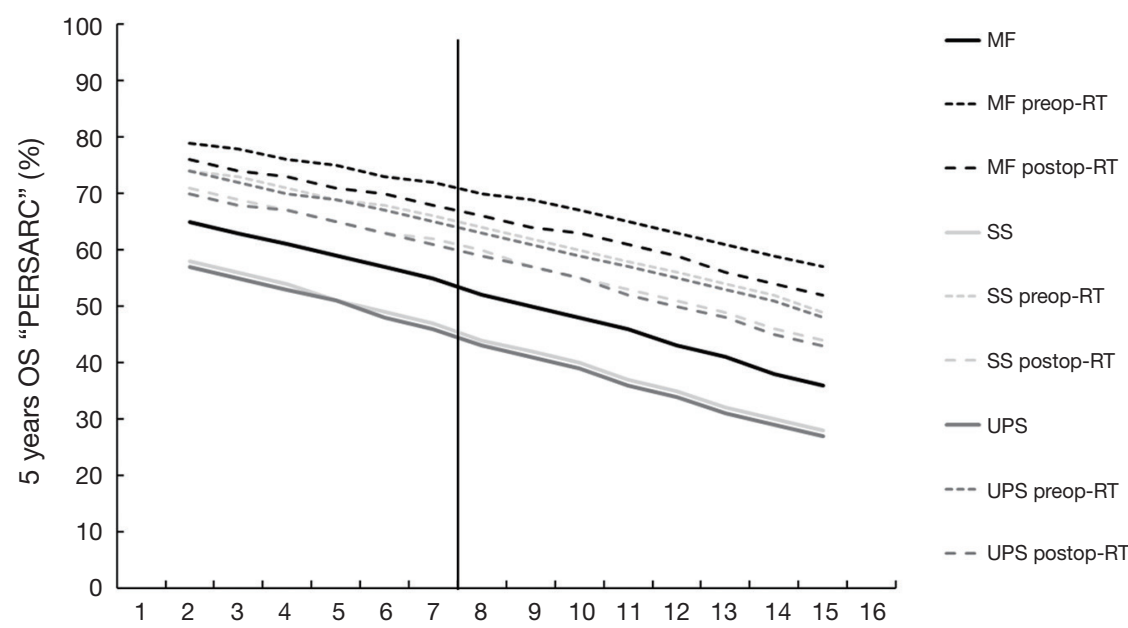

Figure 1 The 5 years OS probability of a 59-year-old sarcoma patient for whom the sarcoma was resected with wide margins (>2 mm) after surgery alone or in combination with perioperative radiotherapy according to the PERSARC-app (4). The vertical line depicts the $7.5 \mathrm{~cm}$ dichotomy as proposed by Johnson et al. (1). OS, overall survival; MF, myxofibrosarcomas; RT, radiotherapy; SS, synovial sarcomas; UPS, undifferentiated pleomorphic sarcoma; PERSARC, Personalised Sarcoma Care.

lumps prediction only based upon dichotomized size and grade, but not upon subtype, depth of tumor location, and both age and size as continuous variables. From figure 1C it can be deducted that in their staging system, stage II and III patients have an on average 5-year survival probability of $77 \%$ and $55 \%$ respectively (data extracted using Engauge Digitizer; available online: http://digitizer.sourceforge.net/).

Focusing on personalization of sarcoma care and prediction of outcome, there are 2 apps available; the "PERSARC" app, based upon the study by van Praag et al. (4) and the "SARCULATOR" app, based upon the study by Callegaro et al. (5). Both models aim to fulfill the need for shared decision-making between patients and physicians, providing a prognostic Personalised Sarcoma Care (PERSARC) model and the nomogram derived SARCULATOR, predicting the cumulative incidences of local recurrence (LR, for PERSARC) and overall survival (OS, in both models) for an individual patient with a highgrade extremity soft tissue sarcoma with specific clinical characteristics and possible treatment modalities at baseline. Available diagnostic subtypes within the PERSARC model are myxofibrosarcomas (MF), malignant peripheral nerve sheet tumors (MPNST), synovial sarcomas (SS), spindle cell sarcomas, undifferentiated pleomorphic sarcoma (UPS) and a rest group of "others". The SARCULATOR is applicable to patients with leiomyosarcoma, dedifferentiated and pleomorphic liposarcomas, myxoid liposarcomas, MPNST, MF, SS, vascular sarcomas, UPS and "others".
In Johnson's system (1) on patients with a median age of 59 years, three of these are also specified being the MF, SS and UPS. Because most of the high-grade sarcomas are predominantly located deep to the fasciae, Johnson's (1), van Praag's (4) and Callegaro's (5) data should be comparable envisioning a prototype 59 years old patient to undergo a resection with wide margin ( $>2 \mathrm{~mm}$ ) alone or in combination with either pre- or post-operative radiotherapy.

Figure 1 shows the 5 years OS probabilities as a function of size of a 59-year-old sarcoma patient, as assessed by "PERSARC", in whom the sarcoma was resected with wide margins and who either were managed with surgery alone (solid lines) or in combination with preoperative radiotherapy (preop-RT, small dashed lines) or postoperative radiotherapy (postop-RT, large dashed lines) and the Johnson and coworkers proposed dichotomy at a size of $7.5 \mathrm{~cm}$. Two observation can be done from this graph. First, MF patients seem to exhibit an on average 10\% more favorable survival as compared to SS and UPS patients with sarcomas of the same size, and second, there is a gradual decline of approximately $2 \%$ OS deficit per centimeter sarcoma size increase regardless of subtype, regardless of mode of management and regardless of the $7.5 \mathrm{~cm}$ dichotomy mark.

Figure 2 shows the 5 years OS probabilities as a function of size of, again, a 59 years old sarcoma patient, as assessed by "SARCULATOR". Two observation can be done from this graph. First, here, the average $7.5 \mathrm{~cm}$ sarcoma size UPS patients seems to exhibit an $18 \%$ more favorable survival 


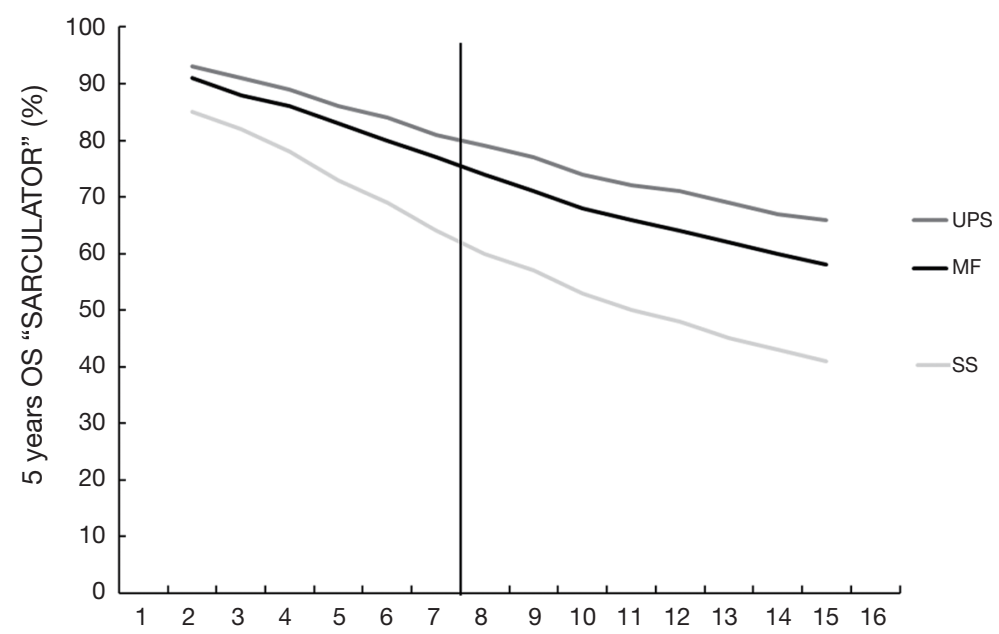

Figure 2 The 5 years OS probability of a 59-year-old sarcoma patient according to the SARCULATOR-app (5). The vertical line depicts the $7.5 \mathrm{~cm}$ dichotomy as proposed by Johnson et al. (1). OS, overall survival; MF, myxofibrosarcomas; SS, synovial sarcomas; UPS, undifferentiated pleomorphic sarcoma; PERSARC, Personalised Sarcoma Care.

Table 1 Comparison of PERSARC and SARCULATOR with respect to OS

\begin{tabular}{|c|c|c|c|c|c|c|c|}
\hline \multirow{2}{*}{$\begin{array}{l}\text { Intentionally } \\
\text { left blank }\end{array}$} & \multirow{2}{*}{ Johnson (1) } & \multicolumn{3}{|c|}{ PERSARC (+ perioperative radiotherapy) (4) } & \multicolumn{3}{|c|}{ SARCULATOR (5) } \\
\hline & & $2-7 \mathrm{~cm}$ & $8-15 \mathrm{~cm}$ & $\begin{array}{c}\text { 5-year OS deficit } \\
\text { per cm }\end{array}$ & $2-7 \mathrm{~cm}$ & $8-15 \mathrm{~cm}$ & $\begin{array}{c}\text { 5-year OS deficit } \\
\text { per cm }\end{array}$ \\
\hline MF & & $70-78 \%$ & $55-68 \%$ & & $77-91 \%$ & $58-74 \%$ & $2 \%$ \\
\hline UPS & $\begin{array}{l}77 \% \text { Gll vs. } \\
55 \% \text { GIII }\end{array}$ & $63-72 \%$ & $46-61 \%$ & $2 \%$ & $81-93 \%$ & $66-79 \%$ & $2 \%$ \\
\hline
\end{tabular}

OS, overall survival; MF, myxofibrosarcomas; SS, synovial sarcomas; UPS, undifferentiated pleomorphic sarcoma; PERSARC, Personalised Sarcoma Care; GII, grade II; GIII, grade III.

as compared to SS patients with sarcomas of the same size, and second, there is a gradual decline of approximately $2 \%$ OS deficit per centimeter sarcoma size increase for UPS and MF, but about up to $3 \%$ for SS, again, regardless of the $7.5 \mathrm{~cm}$ dichotomy mark.

Furthermore, on average, the 5 -year survival outcome as assessed by the SARCULATOR for MF and UPS patients seems to be about $10 \%$ higher as compared to PERSARC. For SS patients, both systems seem to agree. All data are further summarized in Table 1.

In conclusion, Johnson and co-workers are to be congratulated with their manuscript and their proposed staging system. It is simple and can be validated to other databases and staging systems as described in their manuscript. However, as in all systems, by its simplicity it lacks the finesses of details. If those are requested to counsel an individual patient, consulting the PERSARC- and/or
SARCULATOR-apps could be useful.

\section{Acknowledgments}

None.

\section{Footnote}

Conflicts of Interest: The authors have no conflicts of interest to declare.

\section{References}

1 Johnson AC, Ethun CG, Liu Y, et al. A novel, simplified, externally validated staging system for truncal/extremity soft tissue sarcomas: An analysis of the US Sarcoma Collaborative database. J Surg Oncol 2018;118:1135-41. 
2 Edge SB, Byrd DR, Compton CC, et al. AJCC Cancer Staging Manual. 7th edition. New York: Springer, 2010.

3 Amin MB, Edge SB, Greene FL, et al. AJCC Cancer Staging Manual. 8th edition. New York: Springer, 2017.

4 van Praag VM, Rueten-Budde AJ, Jeys LM, et al. A prediction model for treatment decisions in high-grade extremity soft-tissue sarcomas: Personalised sarcoma care
(PERSARC). Eur J Cancer 2017;83:313-23.

5 Callegaro D, Miceli R, Bonvalot S, et al. Development and external validation of two nomograms to predict overall survival and occurrence of distant metastases in adults after surgical resection of localised soft-tissue sarcomas of the extremities: a retrospective analysis. Lancet Oncol 2016;17:671-80.

Cite this article as: Haas RL, van de Sande MAJ. Considering sarcoma staging systems and their implications to personalized care. Chin Clin Oncol 2019;8(Suppl 1):S9. doi: 10.21037/ cco.2019.01.03 